\title{
HOMOGENEOUS STRUCTURES ON KÄHLER SUBMANIFOLDS OF COMPLEX PROJECTIVE SPACES*
}

\author{
by SERGIO CONSOLE and ANNA FINO
}

(Received 9th November 1994)

\begin{abstract}
In this paper we give a differential characterization of homogeneous Kähler submanifolds of complex projective spaces in terms of the existence of a tensor field, the homogeneous structure $S$. We show that for any $m \in M, S_{m}$ determines a unitary representation whose orbit at $m$ is a compact, complete Kähler submanifold which extends $M$. We consider the $U(n) \times U(N-n)\left(n=\operatorname{dim}_{\mathbb{C}} M\right)$ module of the space of these tensors and we find its irreducible factors.
\end{abstract}

1991 Mathematics subject classification: 53C40.

\section{Introduction}

Let $M$ be a Kähler submanifold of $\mathbb{C P}^{N}$. We shall denote by $T M$ and $v M$ the tangent and normal bundle of $M$, respectively; by $\nabla$ and $\nabla^{\perp}$ the Levi-Civita and the normal connection on $M$. $\alpha$ will be the second fundamental form of $M$. We let $J$ be both the complex structure on $M$ and on $\mathbb{C P}^{N}$ (cf. Section 2 below). We denote by $\xi$ the bundle $T M \oplus v M$, i.e. the pull-back on $M$ of the tangent bundle of $\mathbb{C P}^{N}$. The complex structure of $\mathbb{C} \mathbb{P}^{N}$ induces on $M$ a tensor field $J \in T M^{*} \otimes \xi^{*} \otimes \xi$.

Following [24] and [16] (cf. also [5]), we introduce the notion of homogeneous structure on $M$.

Definition. A homogeneous structure on $M$ is a tensor field $S \in T M^{*} \otimes \xi^{*} \otimes \xi$ such that

(1) $T M$ and $v M$ are parallel subbundles of $\xi$ with respect to the connection on $\xi$ given by $\tilde{\nabla}:=\nabla \oplus \nabla^{\perp}-S$.

(2) $\tilde{\nabla}$ is a metric connection.

(3) $\tilde{\nabla} J=0$.

(4) $\tilde{\nabla} \alpha=0$.

(5) $\tilde{\nabla} S=0$.

Our main result is the following differential characterization of homogeneous Kähler submanifolds.

Theorem A. A connected Kähler submanifold $M$ of $\mathbb{C P}^{N}$ is an open subset of a

*Work partially supported by the GNSAGA of CNR and by the MURST of Italy. 
globally homogeneous Kähler submanifold of $\mathbb{C P}^{N}$ if and only if it admits a homogeneous structure $S$.

The proof will be given in Sections 3 and 4.

Note that all examples of homogeneous Kähler submanifold of $\mathbb{C P}^{N}$ can be obtained by means of the Borel-Weil construction ([2], see also Section 2).

Theorem A plays the same role, in the intrinsic case, of a Theorem of K. Sekigawa [20]. Actually the restriction of $\tilde{\nabla}$ to $T M$ is an Ambrose-Singer connection on $M$ compatible with the complex structure $J$. An analogue of Theorem A, for submanifolds of $\mathbb{R}^{N}$ was stated by $C$. Olmos in [16].

A particular case of Theorem A was proved by $H$. Nakagawa and R. Takagi in [14] for Hermitian symmetric Kähler submanifolds of $\mathbb{C} \mathbb{P}^{N}$. These spaces are characterized by the fact that they admit the null tensor as homogeneous structure. Nakagawa and Takagi also classified these submanifolds.

If $S$ is a homogeneous structure on a Kähler submanifold of $\mathbb{C P}^{N}$ and $m=[p] \in M$, the triple $\left(S_{m}, \alpha_{m}, J_{m}\right)$ determines the submanifold uniquely up to isometries (see Theorem 4.1). Hence, a classification of the tensors which can arise as homogeneous structures gives rise to a classification of the extrinsic geometry of the homogeneous Kähler submanifolds of $\mathbb{C P}^{N}$. To this aim, in Section 5 , we will study the space of tensors with the same symmetries as $S_{m}$. Set $V:=T_{m} M, W:=v_{m} M$, if $n=\operatorname{dim} V$ and $h=\operatorname{dim} W$, then one has a (canonically defined) action of $U(n) \times U(h)$ on this space. This $U(n) \times U(h)$ module will be denoted by $\mathscr{D}(V, W)$. We split $\mathscr{D}(V, W)$ into its irreducible components. This decomposition is done following the methods of S. M. Salamon ([19, Chapter 3]; see also [6]). These methods, compared with the ones of Weyl [25] have the advantage that one does not need to prove the irreducibility of the various components, thus avoiding the computation of the quadratic invariants.

Theorem B. The $U(n) \times U(h)$ module $\mathscr{D}(V, W)$ splits as

$$
\mathscr{D}(V, W) \cong \mathscr{T}_{+}(V) \oplus \mathscr{N}(V, W),
$$

where $\mathscr{T}_{+}(V)$ and $\mathcal{N}(V, W)$ are respectively a $U(n)$ module and a $U(n) \times U(h)$-module which correspond to the $V$-component and the $W$-component of a tensor in $\mathscr{D}(V, W)$. Moreover $\mathscr{T}_{+}(V)$ and $\mathcal{N}(V, W)$ have the following decomposition into irreducible factors

$$
\begin{gathered}
\mathscr{T}_{+}(V) \cong 2 \llbracket \lambda_{V}^{1,0} \rrbracket \oplus \llbracket B_{V} \rrbracket \oplus \llbracket\left(\lambda_{V}\right)_{0}^{2,1} \rrbracket \\
\mathcal{N}(V, W) \cong\left(\llbracket \lambda_{V}^{1,0} \rrbracket \otimes\left[\left(\lambda_{W}\right)_{0}^{1,1}\right]\right) \oplus\left(\llbracket \lambda_{V}^{1,0} \rrbracket \otimes \mathbb{R}_{W}\right) .
\end{gathered}
$$

We refer to Section 5 (cf. also [6] and [7]) for the definitions of $\llbracket \lambda_{V}^{1,0} \rrbracket, \llbracket B_{V} \rrbracket \ldots$

Theorem $B$ will be proved in Section 5 as a consequence of Theorems 5.1 and 5.2.

Note that $\mathscr{T}_{+}(V)$ is the Kähler part in the decomposition of homogeneous structures on almost Hermitian manifolds obtained by E. Abbena and S. Garbiero in [1]. More precisely, the space of homogeneous structures on almost Hermitian manifolds $\mathscr{T}(V)$ splits into $\mathscr{T}_{+}(V) \oplus \mathscr{T}_{-}(V)$, where $\mathscr{T}_{+}(V)$ corresponds to the Kähler structures. We 
remark that the components in $\mathscr{T}_{+}(V)$ we obtain here agree with the ones in [1] (there is just a different notation).

The component $\mathcal{N}(V, W)$ obviously comes from the existence of a normal space.

The homogeneous Kähler submanifolds admitting a homogeneous structure in $\mathcal{N}(V, W)$ will be characterized in Section 6.

Moreover, in Section 6 some applications and basic examples will be given. By Lemma 3.1 the homogeneous structure and the second fundamental form at $m$ of an orbit $G \cdot m$ of a unitary representation can be determined in a simple algebraic way. In particular, some geometrical properties of the orbit can be read from the weight lattice of the representation.

\section{Preliminaries}

Let $i:\left(M, g, J_{M}\right) \rightarrow\left(\mathbb{C P}^{N}(c), \bar{g}, J\right)$ be a Kähler submanifold. We denote by $g$ and $\bar{g}$ respectively the Kähler metrics on $M$ and the Fubini-Study metric on $\mathbb{C P}^{N}(c)$ (complex projective space with constant holomorphic sectional curvature $c$ ), and by $J_{M}(J)$ the complex structures of $M\left(\mathbb{C} P^{N}\right)$. Let $\nabla^{M}\left(\nabla^{\mathrm{CP}^{N}}\right)$ be the Levi-Civita connection on $M\left(\mathbb{C} P^{N}\right)$. Then

$$
\begin{aligned}
& i^{*} \bar{g}=g, \quad i^{*} J=J_{M}, \\
& J \nabla^{\mathrm{CP}^{N}}=\nabla^{\mathrm{CPN} J} \text { which implies }\left\{\begin{array}{l}
J_{M} \nabla^{M}=\nabla^{M} J_{M}, \\
\alpha\left(X, J_{M} Y\right)=J \alpha(X, Y),
\end{array}\right.
\end{aligned}
$$

where $X, Y$ are vector fields on $M$ and $\alpha$ is the second fundamental form of $M$.

To simplify the notation, in view of (2.1), we denote by $\langle$,$\rangle both the Kähler metric of$ $M$ and the Fubini-Study metric on $\mathbb{C P}^{N}$ and by the same letters the complex structures on $M$ and $\mathbb{C P}^{N}$.

The rigidity theorem of E. Calabi [3] plays a fundamental role in the study of Kähler submanifolds of $\mathbb{C} \mathbb{P}^{N}$.

Theorem 2.1. (Calabi's Rigidity Theorem). Let $f: M \rightarrow \mathbb{C P}(c)$ and $f^{\prime}: M \rightarrow \mathbb{C} \mathbb{P}^{N^{\prime}}(c)$ be two full Kähler immersions of the same Kähler manifold $M$. Then $N=N^{\prime}$ and there exists a unique holomorphic isometry $\Phi$ of $\mathbb{C P}^{N}$ such that $\Phi f=f^{\prime}$.

As a straightforward corollary, any homogeneous Kähler submanifold is extrinsic homogeneous. Indeed, if $M$ is homogeneous and $G$ is a Lie group acting transitively on $M$ as a group of isometries, any $g \in G$ extends to a unique holomorphic isometry of $\mathbb{C P}^{N}$. Hence $M$ is an orbit of a representation of $G$ in the isometry group of $\mathbb{C P}^{N}$.

There is a classical construction due to Borel and Weil (cf. [2]) which provides all examples of homogeneous Kähler submanifolds of $\mathbb{C P}^{N}$ (cf. [23] and Theorem 2.2 below). Here we sketch such a construction.

Let $G$ be a compact semisimple Lie group and $\Lambda$ a suitable (see [23]) linear combination of the fundamental weights of $G$. Let $\rho$ be the irreducible representation of 
$G$ whose highest weight is $\Lambda$. Denote by $V$ the eigenspace of $\rho$ corresponding to $\Lambda$. Since $\operatorname{dim}_{C} V=1, V$ determines a point $[V] \in \mathbb{C P}^{N}$. The orbit $M=G \cdot[V]$ is a compact homogeneous, simply connected, Kähler submanifold of $\mathbb{C P}^{N}(c)$. The same construction can be done equivalently starting from a simply connected complex simple Lie group $G^{\prime}$ (see [8]; the connection between the two approaches is that the Lie algebra of $G$ is a compact real form of the Lie algebra of $\left.G^{\prime}\right)$.

For example, if $G=S U(n)\left(G^{\prime}=S L(n, \mathbb{C})\right)$ one obtains embeddings of the complex Grassmannian $G(k, n)$ of the $k$ dimensional subspaces of $\mathbb{C}^{n}$. Plücker embeddings of the Grassmannian and the Veronese embedding also arise in the same way (for more details see [8, Section 23.3]).

Theorem 2.2. [23]. Let $f: M \rightarrow \mathbb{C P}^{N}(c)$ be a Kähler immersion of a globally homogeneous Kähler manifold $M$. Then

(1) $M$ is compact and simply connected,

(2) $f$ is an embedding,

(3) $M$ is the orbit in $\mathbb{C P}^{N}$ of the highest weight in an irreducible unitary representation of a compact semisimple Lie group.

Let $m=[p]$ be a point in $\mathbb{C P}^{N}$. We remark that $T_{m} \mathbb{C P}^{N}$ can be identified with the orthogonal complement $\langle m\rangle^{\perp}$ of the plane $\langle m\rangle$ in $\mathbb{C}^{N+1} \cong \mathbb{R}^{2 N+2}$. Since the quotient map

$$
\pi: S^{2 N+1} \subset \mathbb{C}^{N+1} \cong \mathbb{R}^{2 N+2} \rightarrow \mathbb{C P}^{N}
$$

is a Riemannian submersion, using the fundamental equations of submersions [18], we have

Lemma 2.3. Let $\nabla^{\mathrm{CP}^{N}}$ denote the Levi-Civita connection of $\mathbb{C P}^{N}$ (endowed with the Fubini-Study metric) and $\nabla^{\mathbb{R}^{2 N+2}}$ the Levi-Civita connection of $\mathbb{R}^{2 N+2}$ (endowed with the euclidean metric). Then

$$
\nabla_{u}^{C P N} Y=\nabla_{\tilde{u}}^{\mathbb{R}^{2 N}+2} \tilde{Y}-\langle u, Y\rangle p+\langle u, J Y\rangle J p
$$

where $u \in T_{m} \mathbb{C P}^{N}, m=[p]=\pi(p), Y$ is a vector field on $\mathbb{C P}^{N}$ and $\tilde{u}$ and $\tilde{Y}$ are the horizontal lifts of $u$ and $Y$ respectively.

Throughout the paper we will always identify tangent vectors to $\mathbb{C P}^{N}$ with their horizontal lifts.

\section{The canonical homogeneous structure}

Let $\tilde{M} \rightarrow \mathbb{C P}^{N}$ be a homogeneous Kähler submanifold. As remarked in the previous Section, $\tilde{M}$ is the orbit of a point $m=[p] \in \mathbb{C P}^{N}$ in a representation $\rho: G \rightarrow U(N+1)$. We recall how one can define on $\tilde{M}$ a homogeneous structure $S^{c}$, which is canonical as soon 
as a reductive decomposition of the Lie algebra of $G$ is given. It is known (cf. [23], [9]) that if $\tilde{M}$ is an almost Hermitian homogeneous manifold, then there exists a reductive decomposition of $\mathfrak{g}=\mathfrak{h} \oplus \mathrm{m}$ (where $\mathfrak{h}$ is the Lie algebra of the isotropy subgroup at $m$ ) compatible with the complex structure, i.e.

$$
[\mathfrak{h}, \mathrm{m}] \subseteq \mathfrak{m}, \quad \mathfrak{m} \cong T_{m} \tilde{M}
$$

and (via the isomorphism above)

$$
J \mathrm{~m} \subseteq \mathrm{m} \text { and } a d_{\mathrm{h}} J=J a d_{\mathrm{h}}
$$

Imitating a construction due to K. Nomizu (cf. [15]) one can associate to this decomposition a canonical connection $\tilde{\nabla}^{c}$. The difference tensor $S^{c}=\nabla \otimes \nabla^{\perp}-\tilde{\nabla}^{c}$ will be called the canonical homogeneous structure. The connection $\tilde{\nabla}^{c}$ can be characterized by the fact that its geodesics through $m$ are orbits of 1-parameter subgroups, i.e. $\gamma(t)=\exp (t x) \cdot m, x \in \mathrm{m}$ and that the parallel displacement along the geodesics coincides with the differential of the action of $\exp (t x)$. The "only if" part of Theorem $A$ is then straightforward, since one verifies readily that $J, \alpha$ and $S^{c}$ are $\tilde{\nabla}^{c}$-parallel.

Given a representation $\rho: G \rightarrow U(N+1)$ and an orbit of $\rho, \tilde{M}=G \cdot m, S_{m}^{c}$ and the value at $m$ of the second fundamental form can be expressed in terms of the representation of Lie algebras which corresponds to $\rho$. If $Y$ is a tangent vector to $\mathbb{C P}^{N}$ at $m$, let $Y(t):=(\exp (t x))_{* m} \cdot Y$ be the corresponding $\tilde{\nabla}^{c}$ parallel tangent vector field along $\gamma$. Since $\rho(\exp t x): \mathbb{C}^{N+1} \cong\langle m\rangle \oplus\langle m\rangle^{\perp} \rightarrow \mathbb{C}^{N+1} \cong\langle\gamma(t)\rangle \oplus\langle\gamma(t)\rangle^{\perp}$ is linear, we get

$$
(\exp t x)_{\star m} \cdot Y=(\exp t x) \cdot Y
$$

hence

$$
x \cdot Y=\frac{d}{d t_{\mid t=0}}(\exp t x) \cdot Y=\frac{d}{d t_{\mid t=0}}(\exp t x)_{* m} \cdot Y
$$

If $\operatorname{proj}_{\langle m\rangle^{\perp}}$ denotes the projection on $\langle m\rangle^{\perp}$, using Lemma 2.3, we get

$$
\begin{aligned}
\left(S_{m}^{c}\right)_{x} Y & =\left[\nabla_{x}^{C P^{N}}(\exp t x)_{\star m} \cdot Y-\tilde{\nabla}_{x}^{c}(\exp t x)_{* m} \cdot Y\right]_{\mid m} \\
& =\left[\nabla_{x}^{C P^{N}}(\exp t x)_{* m} \cdot Y\right]_{\mid m}=\operatorname{proj}_{\langle m\rangle}\left[\nabla_{x}^{\mathbf{R}^{2 N+2}}(\exp t x)_{* m} \cdot Y\right]_{\mid m} \\
& =\operatorname{proj}_{\langle m\rangle^{\perp}} \frac{d}{d t_{\mid t=0}}(\exp t x)_{* m} \cdot Y=\operatorname{proj}_{\langle m\rangle}(x \cdot Y) .
\end{aligned}
$$

Next we compute the second fundamental form $\alpha_{m}$ of $\tilde{M}$ at $m$. Let $x, y \in T_{m} \tilde{M} \cong m$ (we denote with the same letter the elements of $T_{m} \tilde{M}$ and $m$ ). As above, $y(t):=\exp (t x)_{{ }_{m}} \cdot y$ is a $\left(\tilde{\nabla}^{c}\right.$ parallel) vector field along $\gamma(t):=\exp (t X) \cdot m$. Then

$$
\alpha_{m}(x, y)=\left[\nabla_{x}^{\mathrm{CPN}^{N}} y(t)\right]^{\perp}=\left[\operatorname{proj}_{\langle m\rangle} \nabla_{\tilde{x}}^{\mathbf{R}^{2 N+2}} \tilde{y}(t)\right]^{\perp}=[x \cdot y]^{\perp}
$$


where ${ }^{1}$ denotes the projection on $v_{m} \tilde{M}$. We remark that the isomorphism (3.1) identifies (with abuse of notation) $y$ with $y \cdot m$, so (cf. [11])

$$
\alpha_{m}(x, y)=(x \cdot y \cdot m)^{\perp} .
$$

Hence we have proved the following

Lemma 3.1. With the same notation and assumptions as above,

$$
\begin{gathered}
\left(S_{m}^{c}\right)_{x} Y=\operatorname{proj}_{\langle m\rangle}(x \cdot Y), \\
\alpha_{m}(x, y)=(x \cdot y \cdot m)^{\perp},
\end{gathered}
$$

where $x, y \in \mathrm{m} \cong T_{m} \tilde{M}, Y \in T_{m} \mathbb{C P}^{N}$.

We remark that the restriction of $S^{c}$ to $T M$ determines a homogeneous structure on $M$, whose torsion is given by $T_{x} y=S_{y}^{c} x-S_{x}^{c} y$ (cf. [24]). By (3.4) it follows readily that

$$
T_{x} y=-[x, y] \cdot m \text {. }
$$

\section{The Lie subalgebra associated with a homogeneous structure}

Let $M \rightarrow \mathbb{C} \mathbb{P}^{N}$ be a Kähler submanifold which admits a homogeneous structure $S$ and denote by $\tilde{\nabla}$ the corresponding metric connection. Let $m \in M$ be fixed and consider a curve $\gamma(t)$, with $\gamma(0)=m, \dot{\gamma}(0)=x$. Denote by $\tau_{\gamma(t)}$ the isomorphism of $T_{m} \mathbb{C} \mathbb{P}^{N}=$ $T_{m} M \oplus v_{m} M$ into $T_{y(t)} \mathbb{C P}^{N}=T_{y(t)} M \oplus v_{y(t)} M$ determined by the parallel displacement with respect to $\tilde{\nabla}$ along $\gamma(t)$. Let $\tilde{\gamma}(t)$ be the horizontal lift of $\gamma(t)$ (in the Riemannian submersion $\left.\pi: S^{2 N+1} \rightarrow \mathbb{C} \mathbb{P}^{N}\right)$ such that $\pi(p)=m$. We identify $\mathbb{C}^{N+1}$ with $T_{m} \mathbb{C} \mathbb{P}^{N} \oplus \mathbb{R}\{p\}$ $\oplus \mathbb{R}\{J p\}$. For any $t$, there exists a unique unitary transformation $F_{t} \in U(N+1)$ such that

$$
F_{t}(p)=\tilde{\gamma}(t), \quad F_{t}(J p)=J \tilde{\gamma}(t), \quad\left(F_{t}\right)_{s m}=\tau_{\gamma(t)} .
$$

This gives a one parameter subgroup of $U(N+1)$, or, in other words, a curve based at the identity in $U(N+1)$. Hence the tangent vector at $I$ to the curve $F$, is an element $\varphi_{x}$ of the Lie algebra $u(N+1)$.

To simplify the notation, we denote by $\alpha, A$ and $\bar{S}$ the value at $m$ of the second fundamental form, the shape operator and homogeneous structure, respectively. Using Lemma 2.3 and the fundamental equations of an immersion, a straightforward computation shows that $\varphi_{x}$ is described by

$$
\varphi_{x}: \begin{cases}p \mapsto & x \\ J p \mapsto & J x \\ v \mapsto & \bar{S}_{x} v+\alpha\left(x, v^{\top}\right)-A_{v^{1}} x+\langle x, J v\rangle J p-\langle x, v\rangle p\end{cases}
$$


where $v \in T_{m} \mathbb{C P} P^{N}$ and $v^{\top}\left(v^{\perp}\right)$ is the orthogonal projection of $v$ on $T_{m} M\left(v_{m} M\right)$.

Let $\tilde{R}_{x y}$ be the curvature tensor of $\tilde{\nabla}$, computed at $m$. By the Ambrose-Singer holonomy theorem, the Lie algebra of the holonomy group of $\tilde{\nabla}$ is generated by the elements of $u(N+1)$ which act as follows

$$
\tilde{R}_{x y}: \begin{cases}p \mapsto & 0 \\ J p \mapsto & 0 \\ v \mapsto & \tilde{R}_{x y} v .\end{cases}
$$

Moreover to the Kähler form of $\mathbb{C P}^{N}$ correspond the operators

$$
\rho_{x y}: \begin{cases}p \mapsto & \langle x, J y\rangle J p \\ J p \mapsto & -\langle x, J y\rangle p \\ v \mapsto & \langle x, J y\rangle J v .\end{cases}
$$

The operators $\varphi_{x}, \rho_{y z}, \tilde{R}_{u t}\left(x, y, z, u, t \in T_{m} M\right)$ span a Lie subalgebra $\mathfrak{g}$ of $\mathfrak{u}(N+1)$. Indeed, the Gauss, Ricci and Codazzi equations and the definition of homogeneous structure imply that the Lie brackets of these operators are:

$$
\begin{gathered}
{\left[\varphi_{u}, \varphi_{v}\right]=\varphi_{\bar{S}_{u v}-\bar{S}_{v u}}+\tilde{R}_{u v}+2 \rho_{u v},} \\
{\left[\tilde{R}_{u v}, \varphi_{z}\right]=\varphi_{\tilde{R}_{u v}}} \\
{\left[\tilde{R}_{u v}, \tilde{R}_{z w}\right]=\tilde{R}_{\bar{R}_{u v z w}}+\tilde{R}_{z \tilde{R}_{u v} w}} \\
{\left[\rho_{x y}, A\right]=0, \text { for any } A \in \mathfrak{g} .}
\end{gathered}
$$

Theorem 4.1. Let $M$ be a Kähler submanifold of $\mathbb{C P}^{N}$ that admits a homogeneous structure $S$ and let $G$ be the unique connected Lie subgroup of $U(N+1)$ whose Lie algebra is $\mathfrak{g}$. The orbit of $m \in \mathbb{C P}^{N}, \tilde{M}:=G \cdot m$ is a complete Kähler submanifold of $\mathbb{C P}^{N}$ that extends $M$ (up to isometries).

In particular the values of $S, \alpha$ and $J$ at $m$ uniquely determine $\tilde{M}$ (up to isometries).

Proof. The Lie algebra $\mathfrak{g}$ admits the reductive decomposition $\mathfrak{g}=\mathfrak{h} \oplus \mathrm{m}$, where

$$
\mathrm{h}=\operatorname{span}\left\{\tilde{R}_{u v}, \rho_{w z}, u, v, w, z \in T_{m} M\right\}, \quad \mathrm{m}=\operatorname{span}\left\{\varphi_{u}, u \in T_{m} M\right\} .
$$

Note that $\mathrm{m} \cong T_{m} \tilde{M}$ and that

$$
T_{m} \tilde{M}=\operatorname{span}\left\{\varphi_{u} \cdot m, u \in T_{m} M\right\} \cong T_{m} M
$$

Let $\tilde{\nabla}^{c}$ be the canonical connection on $\tilde{M}$ associated to the reductive decomposition 
above. By Lemma 3.1 we get that $S_{m}^{c}=S_{m}$ and the second fundamental form at $m$ of $M$ and $\tilde{M}$ coincide.

Remark moreover that the complex structures of $M$ and $\tilde{M}$ at $m$ coincide via the isomorphisms $T_{m} M \cong m \cong T_{m} \tilde{M}$. A very similar argument as the one in the proof of Proposition 2.1 in [17], shows that there exists an isometry $F: \mathbb{C} \mathbb{P}^{N} \rightarrow \mathbb{C P}^{N}$ such that $F(m)=m, F(M) \subseteq \tilde{M}$.

This proves Theorem 4.1, which is the "if" part of Theorem A.

\section{Algebraic decomposition of the space of the homogeneous structures}

Let $i: M \mapsto \mathbb{C P}^{N}$ be a Kähler submanifold. Let $m \in M$, put $V:=T_{m} M, W=v_{m} M$, with $\operatorname{dim}_{\mathrm{R}} V=\operatorname{dim}_{\mathbb{R}} M=2 n$ and $\operatorname{dim}_{\mathbb{R}} W=\operatorname{codim}_{\mathrm{R}} M=2 h$. Using the hermitian metrics on $V$ and $W$ we shall make no distinction between covariant and contravariant tensors. In particular a homogeneous structure shall be considered as a tensor $D_{x Y Z}$ on $V^{*} \otimes$ $(V \oplus W)^{*} \otimes(V \oplus W)^{*}$. By definition of homogeneous structure, the symmetries of $D$ are

$$
\begin{gathered}
D_{x Y Z}=-D_{x Z Y}, \\
D_{x y N}=0, \\
D_{x Y Z}=D_{x J Y J Z},
\end{gathered}
$$

where $x, y$ are vectors in $V, N$ belongs to $W, X, Y, Z$ are in $V \oplus W$. Hence

$$
\begin{gathered}
\mathscr{D}(V, W)=\left\{D \in V^{*} \otimes(V \oplus W)^{*} \otimes(V \oplus W)^{*} / D_{x Y Z}=-D_{x Z Y}, D_{x y N}=0,\right. \\
\left.D_{x Y Z}=D_{x J Y J Z}, x, y \in V, N \in W, Y, Z \in V \oplus W\right\}
\end{gathered}
$$

is the space of tensors having the same symmetries as the homogeneous structures on Kähler submanifolds of $\mathbb{C P}^{N}$.

The inner product on $V$ induces canonically an inner product on $\mathscr{D}(V, W)$ and determines an $U(n)$-equivariant isomorphism $V \cong V^{*}$ and an $U(h)$-equivariant isomorphism $W \cong W^{*}$.

The standard representation of $U(n) \times U(h)$ (regarded as a subgroup of $U(n+h)$ ) gives rise to a representation of $U(n)$ on $V$ and of $U(h)$ on $W$ and thus a representation of $U(n) \times U(h)$ on $\mathscr{D}(V, W)$ defined by

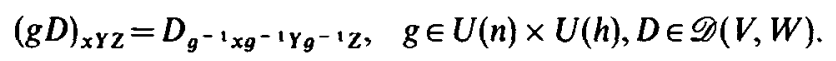

It follows immediately that the above representation is completely reducible.

Because of $(5.1) \mathscr{D}(V, W)$ is $U(n) \times U(h)$-equivariantly included into

$$
V \otimes \wedge^{2}(V \otimes W) \cong V \otimes\left(\wedge^{2} V \oplus(V \otimes W) \oplus \wedge^{2} W\right)
$$


By (5.2) there is an $U(n) \times U(h)$-equivariant inclusion

$$
\mathscr{D}(V, W) \subseteq\left(V \otimes \wedge^{2} V\right) \oplus\left(V \otimes \wedge^{2} W\right)
$$

It is clear that $(J,\langle\rangle$,$) defines Kähler structures on V$ and $W$. The complexification of the dual spaces $V^{*}$ and $W^{*}$ are

$$
V^{*} \otimes_{\mathbf{R}} \mathbb{C}=\lambda_{V}^{1,0} \oplus \lambda_{V}^{0,1}, \quad W^{*} \otimes_{\mathbf{R}} \mathbb{C}=\lambda_{W}^{1,0} \oplus \lambda_{W}^{0,1},
$$

where $\lambda_{V}^{1.0}$ is the annihilator of the space of complex tangent spaces vectors of the form $X+i J X$ and $\overline{\lambda_{v}^{1,0}}=\lambda_{v}^{0,1}$ is its conjugate (the same for $\lambda_{\dot{W}}^{i, j}$ ). In the sequel we shall deal mainly with $V$ and it shall be understood that what we say holds for $W$, too. We refer to [19], [7] and [6] for more details.

The $(p+q)$-th exterior power of $\bar{V}^{*}$ contains a subspace $\lambda_{V}^{p, q}$ (which is isomorphic to $\left.\wedge^{p} \lambda_{V}^{1,0} \otimes \wedge^{q} \lambda_{V}^{0,1}\right)$ consisting of the so-called forms of type $(p, q)$. Both $\lambda_{V}^{p, q} \oplus \lambda_{V}^{q, p},(p \neq q)$ and $\lambda_{i}^{p, p}$ are the complexifications of real vector spaces which we denote by $\llbracket \lambda_{v}^{p} q \rrbracket$ and $\left[\lambda p^{p}\right]$ respectively, so that

$$
\llbracket \lambda_{V}^{p, q} \rrbracket \otimes_{\mathbb{R}} \mathbb{C}=\lambda_{V}^{p, q} \oplus \lambda_{V}^{q, p}, \quad(p \neq q)
$$

and

$$
\left[\lambda_{V}^{p, p}\right] \otimes_{\mathbf{R}} \mathbb{C}=\lambda_{i}^{p} p
$$

The space of 2-forms decomposes as

$$
\left.\wedge^{2} \vec{V}^{*}=\left[\lambda_{v}^{1,1}\right] \oplus \llbracket \lambda_{V}^{2,0}\right]
$$

Here $\left[\lambda_{V}^{1}, 1\right]$ equals the subspace of 2-forms $\sigma$ for which $\sigma(X-i J X, Y-i J Y)=0$ or equivalently $\sigma(J X, J Y)=\sigma(X, Y)$, for all $X, Y \in V \oplus W$. Moreover, $\sigma \in \llbracket \lambda_{V}^{2}{ }^{0} \rrbracket$ if and only if $\sigma(J X, J Y)=-\sigma(X, Y)$, for all $X, Y \in V \oplus W$. One may identify $\wedge^{2} V$ with the Lie algebra $\operatorname{so}(2 n),\left[\lambda_{v}^{1,1}\right]$ with the subalgebra $u(n)$ and $\left[\left(\lambda_{0}\right)_{v}^{1,1}\right]$ with $\mathfrak{s u}(n)$.

More generally, let $\omega_{V}$ be the Kähler form on $V$, i.e. $\omega_{V}=-i \sum_{\alpha} d z^{\alpha} \wedge d \bar{z}^{\alpha}$. Wedging with $\omega_{V}$ determines an $U(n)$-equivariant mapping $L_{V}: \lambda_{V}^{p-1, q-1} \rightarrow \lambda_{V}^{p, q} .\left(\lambda_{0}\right)_{V}^{p, q}$ is defined to be the orthogonal complement of the image of $L_{V}$ with respect to the induced Hermitian metric. It is well known that the complex $U(n)$-modules $\left(\lambda_{V}\right)_{0}^{p, q}$ are irreducible.

Finally, we denote by $\mathbb{R}_{V}$ the module $\left[\lambda_{V}^{0,0}\right]$, i.e. the trivial representation on $V$.

Note now that, by $(5.1), \ldots,(5.3)$ we have the $U(n) \times U(h)$-equivariant isomorphism

$$
\mathscr{D}(V, W) \cong\left(V \otimes\left[\lambda_{V}^{1,1}\right]\right) \oplus\left(V \otimes\left[\lambda_{W}^{1}{ }^{1}\right]\right)
$$

Set

$$
\mathscr{T}_{+}(V):=V \otimes\left[\lambda_{V}^{1,1}\right] \quad \mathcal{N}(V, W):=V \otimes\left[\lambda_{W}^{1,1}\right]
$$

Every $D \in \mathscr{D}(V, W)$ splits into two components, i.e. 


$$
D=T+N, \quad T \in \mathscr{T}_{+}(V), \quad N \in \mathcal{N}(V, W) .
$$

Let $B_{V}$ denote the kernel of the antisymmetrization $\lambda_{V}^{1,0} \otimes\left(\lambda_{V}\right)_{0}^{1,1} \mapsto \lambda_{V}^{2,1}$.

Theorem 5.1. There is an isomorphism of $U(n)$-modules

$$
\mathscr{T}_{+}(V) \cong 2 \llbracket \lambda_{V}^{1,0} \rrbracket \oplus \llbracket B_{V} \rrbracket \oplus \llbracket\left(\lambda_{V}\right)^{2,1} \rrbracket
$$

Proof. We have $V=\llbracket \lambda_{V}^{1,0} \rrbracket$, so

$$
\begin{aligned}
\mathscr{T}_{+}(V) & \cong V \otimes\left[\lambda_{V}^{1,1}\right] \\
& \cong \llbracket \lambda_{V}^{1,0} \rrbracket \otimes\left(\left[\left(\lambda_{V}\right)_{0}^{1,1}\right] \oplus \mathbb{R}_{V}\right) \\
& \cong \llbracket \lambda_{V}^{1,0} \rrbracket \otimes\left[\left(\lambda_{V}\right)_{0}^{1,1}\right] \oplus \llbracket \lambda_{V}^{1,0} \rrbracket \\
& \cong 2 \llbracket \lambda_{V}^{1,0} \rrbracket \oplus \llbracket B_{V} \rrbracket \oplus \llbracket\left(\lambda_{V}\right)_{0}^{2,1} \rrbracket
\end{aligned}
$$

$\llbracket \lambda_{V}^{1}, 0 \rrbracket, \llbracket B_{V} \rrbracket, \llbracket\left(\lambda_{V}\right)_{0}^{2,1} \rrbracket$ are irreducible $U(n)$-modules. In fact, in Weyl's correspondence $\llbracket \lambda_{V}^{1}, 0 \rrbracket, \llbracket B_{V} \rrbracket$ and $\llbracket\left(\lambda_{V}\right)_{0}^{2,1} \rrbracket$ are associated to the dominant weights $(1,0, \ldots, 0)$, $(2,0, \ldots, 0,-1)$ and $(1,1,0, \ldots, 0,-1)$, respectively.

See [1] for the expressions of the projections of $D \in \mathscr{T}_{+}(V)$ on the various factors.

Theorem 5.2. There is an isomorphism of $U(n) \times U(h)$-modules

$$
\mathcal{N}(V, W) \cong\left(\llbracket \lambda_{V}^{1}, 0 \rrbracket \otimes\left[\left(\lambda_{W}\right)_{0}^{1,1}\right]\right) \oplus\left(\llbracket \lambda_{V}^{1,0} \rrbracket \otimes \mathbb{R}_{W}\right)
$$

\section{Proof.}

$\mathcal{N}(V, W) \cong V \otimes\left[\lambda_{W}^{1,1}\right] \cong V \otimes\left(\left[\left(\lambda_{W}\right)_{0}^{1,1}\right] \oplus \mathbb{R}_{W}\right) \cong\left(\llbracket \lambda_{V}^{1,0} \rrbracket \otimes\left[\left(\lambda_{W}\right)_{0}^{1,1}\right]\right) \oplus\left(\llbracket \lambda_{V}^{1,0} \rrbracket \otimes \mathbb{R}_{W}\right)$.

Now $\llbracket \lambda_{V}^{1,0} \rrbracket$ is an irreducible $U(n)$ module, $\left[\left(\lambda_{W}\right)_{0}^{1,1}\right]$ and $\mathbb{R}_{W}$ are irreducible $U(h)$ modules. The result follows from the fact that the tensor product of an irreducible $U(n)$ module and an irreducible $U(h)$ module is an irreducible $U(n) \times U(h)$ module. Let

Finally we determine the projection of $D \in \mathcal{N}(V, W)$ on the two irreducible factors.

$$
c_{12}(D)(x):=\sum_{\alpha}\left\langle D_{x} e_{\alpha}, J e_{\alpha}\right\rangle
$$

where $e_{\alpha}$ is an orthonormal basis of $W$. Then

$$
\begin{gathered}
\llbracket \lambda_{V}^{1,0} \rrbracket \otimes\left[\left(\lambda_{W}\right)_{0}^{1,1}\right]=\left\{D \in \mathcal{N}(V, W) / c_{12}(D)=0\right\}, \\
\llbracket \lambda_{V}^{1,0} \rrbracket \otimes \mathbb{R}_{W}=\left\{D \in \mathcal{N}(V, W) /\left\langle D_{x} Y, Z\right\rangle=-\frac{1}{n} c_{12}(D)(x)\langle Y, J Z)\right\} .
\end{gathered}
$$




\section{Examples}

6.1. 2-symmetric Kähler submanifolds of $\mathbb{C P}^{N}$.

Let $M \rightarrow \mathbb{C P}^{N}$ be a complex submanifold of $\mathbb{C P}^{N}$. We recall that $M$ is then a Kähler submanifold.

Imitating [12] and [13] we give the following:

Definition 6.1. $M$ is a 2-symmetric Kähler submanifold of $\mathbb{C P}^{N}$ if there exists a family $\left\{\sigma_{m}\right\}_{m \in M}$ of involutive isometries of $\mathbb{C}^{N}$ which leave the submanifold $M$ invariant, such that any $m \in M$ is an isolated fixed point of $\sigma_{m \mid M}$, and for any $m, q \in M, \sigma_{m} \cdot \sigma_{q}=\sigma_{r} \cdot \sigma_{m}$, where $r=\sigma_{m}(q)$.

Note that the definition implies that $M$ is a symmetric Kähler manifold. Indeed, $\left\{\sigma_{m \mid M}\right\}_{m \in M}$ is a family of symmetries of $M$.

We recall [21] that the $k$-osculating space to $M$ at a point $m \in M, \stackrel{k}{O}_{m}$, is the span of

$$
\left\{X_{1}, \nabla_{X_{1}}^{\mathrm{CPN}^{N}} X_{2}, \ldots, \nabla_{X_{1}}^{\mathrm{CP}^{N}} \nabla_{X_{2}}^{\mathrm{CP}^{N}} \ldots \nabla_{X_{k-1}}^{\mathrm{CP}^{N}} X_{k}\right\}
$$

computed at $m$, where $X_{i}$ are vector fields on $M$. The orthogonal complement $\stackrel{k}{N}_{m}$ of $\stackrel{k}{O}_{m}$ in ${ }^{k+1} O_{m}$ is called the $k$-normal space. If the dimension of every $\stackrel{k}{o}_{m}$ does not depend on $m$, the $k$-osculating and $k$-normal bundles $\stackrel{k}{O}$ and $\stackrel{k}{N}$ are defined. Their fibres at a point $m$ are $\stackrel{k}{O}_{m}$ and $\stackrel{k}{N}_{m}$ respectively. If $\xi \in \stackrel{k}{N}$, then, for any vector field $X$ on $M, \nabla_{X} \xi \in \stackrel{k}{N}{ }^{1} \oplus \stackrel{k}{N} \oplus$ $\stackrel{k+1}{N}$. The higher order second fundamental forms ${ }_{B}^{k}$ at $m$ are defined inductively by

$$
\begin{aligned}
& \stackrel{1}{B}\left(x_{0}, x_{1}\right):=\alpha\left(x_{0}, x_{1}\right), \\
& \stackrel{k}{B}\left(x_{0}, \ldots, x_{k}\right):=\operatorname{proj}_{\left(N_{m} \oplus \ldots \oplus^{k} \hat{N}_{m}^{\prime}\right)^{l}} \nabla_{x_{0}}^{C^{C N}{ }^{k-1}} B^{1}\left(X_{1}, \ldots, X_{k}\right)
\end{aligned}
$$

where $X_{i}$ are vector fields extending $x_{i}$. A metric connection on any $k$-normal space is given by

$$
\stackrel{k}{\nabla}_{X} \xi:=\operatorname{proj}_{\hat{N}} \nabla_{X}^{C^{N}} \xi
$$

Lemma 6.1. If $M$ is a Kähler submanifold of $\mathbb{C P}^{N}$ then $J \stackrel{k}{N} \subseteq \stackrel{k}{N}$

Proof. By induction on $k$. For $k=1$ we remark that $\alpha(x, J y)=J \alpha(x, y)$ (cf. (2.1)) implies $\quad J \stackrel{1}{N} \subset \stackrel{1}{N}$. Suppose $\quad J^{k-1} \subset^{k-1} N^{1}$ and that $\quad{ }_{B}^{k}\left(x_{0}, \ldots, J x_{l}, \ldots, x_{k-1}\right)=$ $J^{k-1}{ }^{-1}\left(x_{0}, \ldots, x_{l}, \ldots, x_{k-1}\right)$. Then 


$$
\begin{aligned}
& \left.\stackrel{k}{B}\left(x_{0}, \ldots, J x_{l}, \ldots, x_{k}\right)=\operatorname{proj}_{\left(\mathcal{N}_{m} \oplus \ldots \oplus \mathcal{N}^{\prime}\right)^{2}} \nabla_{x_{0}}^{\mathrm{CPN}^{k}{ }^{k-1}}\left(X_{1}, \ldots, J X_{l}, \ldots, X_{k}\right)\right) \\
& \left.=\operatorname{proj}_{\left(\stackrel{\circ}{m}_{m} \oplus \ldots \oplus^{*} N_{m}^{\prime}\right)^{1}} \nabla_{x_{0}}^{C P^{N}} J^{k-1} B^{-1}\left(X_{1}, \ldots, X_{l}, \ldots, X_{k}\right)\right)
\end{aligned}
$$

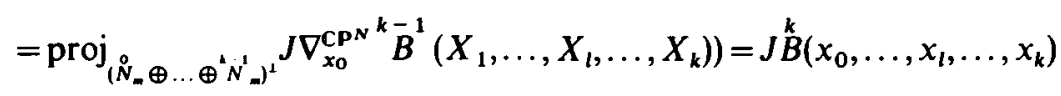

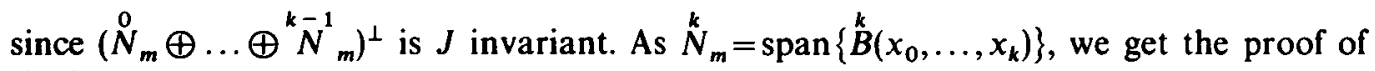
the lemma.

A direct consequence of Lemma 6.1 and $\nabla^{\mathrm{CP}^{N}} J=0$ is

Lemma 6.2. If $M$ is a Kähler submanifold of $\mathbb{C P}^{N}$, then $\stackrel{k}{\nabla} J=0$.

Using Lemma 6.2 and the same techniques as in [4] (cf. also [22]) one can then prove

Theorem 6.3. $\quad M$ is a 2-symmetric Kähler submanifold of $\mathbb{C P}^{N}$ if and only if

$$
\stackrel{k}{\nabla} B=0
$$

Let $\bar{\nabla}^{\perp}$ the metric connection on $v(M)$ given by

$$
\bar{\nabla}^{\perp}:=\sum_{k \geqq 1} \stackrel{k}{\nabla}
$$

A straightforward computation shows that

Lemma 6.4. $M$ is a 2-symmetric Kähler submanifold of $\mathbb{C P}^{N}$ if and only if $\bar{S}:=$ $\nabla \oplus \nabla^{\perp}-\nabla \oplus \bar{\nabla}^{\perp}$ is a homogeneous structure on $M$.

By Lemma 6.1 it is easy to see that $c_{12}(\bar{S})=0$, so $\bar{S} \in \llbracket \lambda_{V}^{1,0} \rrbracket \otimes\left[\left(\lambda_{W}\right)_{0}^{1,1}\right]$. Hence a 2-symmetric Kähler submanifold admits a homogeneous structure belonging to $\llbracket \lambda_{v}^{1}{ }^{0} \rrbracket \otimes$ $\left[\left(\lambda_{W}\right)_{0}^{1.1}\right]$.

On the other hand, suppose that a Kähler submanifold $M$ admits a homogeneous structure in $\mathcal{N}(V, W)$ and let $\mathfrak{g}=\mathfrak{h} \oplus \mathrm{m}$ be the Lie subalgebra of $\mathfrak{u}(N+1)$ constructed in Section 4. By Lemma 3.1 in [10], if $x \in m$, then

$$
x \cdot \stackrel{k}{N}_{m} \subset \stackrel{-1}{N}_{m} \oplus \stackrel{k+1}{N}_{m}
$$


(note that our $\stackrel{k}{N}_{m}$ coincide with the modules $V_{k}$ in $[10]$ for $k \geqq 1$ ). In the same vein as in the proof of Lemma 3.1, we have

$$
\left(\stackrel{k}{\nabla}_{x}^{k}\right)\left(x_{0}, \ldots, x_{k}\right)=\operatorname{proj}_{\hat{N}_{k}}\left[x \cdot \stackrel{k}{B}\left(x_{0}, \ldots, x_{k}\right)\right]=0,
$$

by (6.1). Applying Theorem 6.3 we get that $M$ is 2 -symmetric. Hence

Theorem 6.5. $M$ is a 2-symmetric Kähler submanifold of $\mathbb{C P}^{N}$ if and only if it admits a homogeneous structure belonging to $S \in\left[\lambda_{V}^{1,0}\right] \otimes\left[\left(\lambda_{W}\right)_{0}^{1,1}\right]$.

Remark. The above results imply that, if $M$ admits a homogeneous structure belonging to $\mathcal{N}(V, W)$, then the $\left.\llbracket \lambda_{V^{1}}, 0\right] \otimes \mathbb{R}_{W}$ factor can be eliminated. Indeed, if $M$ has a homogeneous structure in $\mathcal{N}(V, W)$, then it is 2 -symmetric and by Lemma 6.4 it has a homogeneous structure in $\llbracket \lambda_{V}^{1}, 0 \rrbracket \otimes\left[\left(\lambda_{W}\right)_{0}^{1,1}\right]$.

\subsection{Illustration of some examples.}

We recall that any homogeneous Kähler submanifold is an orbit in a unitary representation of compact Lie group $G$. Hence, using Lemma 3.1, one can recover the canonical homogeneous structure and the second fundamental form at $m$ of the orbit $G \cdot m$ starting from the weight lattice of the Lie algebra $\mathfrak{g}$ of $G$.

Example 6.1. Let $\mathfrak{g}=\mathfrak{s u}(2)$. Let $\rho_{\alpha}$ be the representation with highest weight $\alpha \in \mathbb{Z}_{+}$. Let $v_{\alpha}$ be a weight vector relative to $\alpha$ and consider the orbit of [ $v_{\alpha}$ ]. The isotropy subgroup at $\left[v_{\alpha}\right]$ is isomorphic to $U(1)$ and the Kähler submanifold is $\mathbb{C P}^{1} \rightarrow \mathbb{C P}^{N(\alpha)}$. It is easy to see that if $u \in \stackrel{k}{N}_{[p]}$, then $S_{m Y}^{c} u \in \stackrel{k+1}{N}_{m}$ (where $Y$ is a root vector relative to the root -2 ). This shows that $\mathbb{C P}^{1} \rightarrow \mathbb{C P}^{N(\alpha)}$ is a 2 -symmetric Kähler submanifold.

Example 6.2. Let $g=s u(3)$ and $\Lambda$ be a weight which lies in the fundamental Weyl chamber (including its walls). We consider the representation having $\Lambda$ as highest weight.

(a) Let $\Lambda$ belong to a wall of the fundamental Weyl chamber (which one is immaterial). Let $v$ be an eigenvector relative to $\Lambda$ and consider the orbit of $[v]$. The isotropy subgroup at $[v]$ is isomorphic to $S(U(1) \times U(2))$ and the orbit is $\mathbb{C P}^{2}$. Drawing the picture of the weight lattice one can visualize the normal spaces. In particular one can see that the orbit is a 2-symmetric Kähler submanifold.

(b) Let $\Lambda$ lie in the interior of the fundamental Weyl chamber. In this case the orbit is not symmetric. (Indeed the orbit is the manifold of all flags in $\mathbb{C}^{3}$; cf. [8, page 383].) To see this directly from the weight lattice diagram, using homogeneous structures, we now give an explicit example. To this aim it is simpler to consider the complexified Lie algebra $\operatorname{sl}(3, \mathbb{C})$ (cf. what remarked in Section 2). Let $L_{i}$ denote the functional

$$
L_{i}:\left(\begin{array}{lll}
a_{1} & 0 & 0 \\
0 & a_{1} & 0 \\
0 & 0 & a_{3}
\end{array}\right) \mapsto a_{i},
$$


and choose as positive simple roots $L_{1}-L_{2}$ and $L_{2}-L_{3}$. We now take the irreducible representation $\rho$ having highest weight $2 L_{1}-L_{3}$. Let $v$ belong to the weight space of $2 L_{1}-L_{3}$ and consider the orbit $M$ of $v$. One can readily see from the weight lattice diagram that the tangent space is (complex) three dimensional. Let $X \in \mathfrak{s l}(3, \mathbb{C})$ lie in the root space of $L_{3}-L_{2}, Y$ in the root space of $L_{2}-L_{1}$ and set $Z:=[X, Y]$ (thus $Z$ lies in the root space of $L_{3}-L_{1}$ ). Then $x:=\rho(X) v, y:=\rho(Y) v$ and $z:=\rho(Z) v$ are respectively in the weight space of $2 L_{1}-L_{2},-2 L_{3}$ and $L_{1}$ and they span the tangent space of $M$ at $[v]$. By (3.6) in Section 3 we have

$$
T_{x} y=-\rho([X, Y]) v=-\rho(Z) v=-z \neq 0
$$

This shows that the canonical homogeneous structure on $M$ has non-vanishing torsion, which clearly implies that the orbit is not symmetric.

Acknowledgements. We wish to thank Jürgen Berndt and Sergio Garbiero for their useful advice. We are also grateful to the referee for his helpful comments.

\section{REFERENCES}

1. E. Abbena and S. Garbiero, Almost Hermitian homogeneous structures, Proc. Edinburgh Math. Soc. 31 (1988), 375-395.

2. A. Borel et A. WeIL, Représentations linéares et espaces homogènes kählériens des groupes de Lie compacts (Séminaire Bourbaki, exposé by J. P. Serre, 1954).

3. E. CaLABI, Isometric imbedding of complex manifolds, Ann. of Math. 58 (1953), 1-23.

4. A. Carfagna D'Andrea, R. Mazzocco and G. Romani, Some characterizations of 2-symmetric submanifolds in spaces of constant curvature, Czechoslovak Math. J. 44 (119) (1994), 691-711.

5. S. Console, Infinitesimally homogeneous submanifolds of Euclidean spaces, Ann. Global Anal. Geom. 12 (1994), 313-334.

6. M. Falcitelli, A Farinola and S. Salamon, Almost hermitian geometry, Diff. Ann. Global Anal. Geom. Appl. 4 (3) (1994), 259-282.

7. A. Fino, Almost contact homogeneous structures, Boll. Un. Mat. Ital., to appear.

8. W. Fulton and J. Harris, Representation Theory (Springer, Berlin, New York, 1991).

9. A. Gray, Homogeneous almost Hermitian manifolds, Rend. Sem. Mat. Univ. Politec. Torino, Fasc. Spec. (1983), 17-58.

10. O. IKaWA, Harmonic mappings, minimal and totally geodesic immersions of compact Riemannian homogeneous spaces into Grassmann manifolds, Kodai Math. J. 16 (1993), 295-305.

11. E. Kelly, Tight equivariant imbeddings of symmetric spaces, J. Diff. Geom. 7 (1972), 535-548.

12. O. Kowalski, Generalized Symmetric Spaces (Lecture Notes in Math. 805, Springer, Berlin, Heidelberg, New York, 1980).

13. O. KowalsKı and I. KULıCH, Generalized symmetric submanifolds of Euclidean Spaces, Math. Ann. 277 (1987), 67-78. 
14. H. Nakagawa and $R$. Tagaki, On locally symmetric Kähler submanifolds in a complex projective space, J. Math. Soc. Japan 28 (1976), 638-667.

15. K. Nomizu, Invariant affine connections on homogeneous spaces, Amer. J. Math. 76 (1954), 33-65.

16. C. Otmos, Isoparametric submanifolds and their homogeneous structures, J. Differential Geom. 38 (1993), 225-234.

17. C. Olmos and C. Sánchez, A geometric characterization of the orbits of s-representations, Differential J. Reine Angew. Math. 420 (1991), 195-202.

18. B. O'Neill, The fundamental equations of a submersion, Michigan Math. J. 13 (1966), 459-469.

19. S. M. SAlamon, Riemannian Geometry and holonomy groups (Pirman research notes, Longman, Harlow, Essex, UK, 1989).

20. K. Sekigawa, Notes on homogeneous almost Hermitian manifolds, Hokkaido Math. J. 7 (1978), 206-213.

21. M. SpIVAK, A Comprehensive Introduction to Differential Geometry, IV (Publish or Perish, Wilmington, Delaware, 1979).

22. W. Strübing, Symmetric submanifolds of Riemannian manifolds, Math. Ann. 245 (1979), $37-44$.

23. M. TAKEUCHI, Homogeneous Kähler submanifolds in complex projective spaces, Japan $J$. Math. 4 (1) (1978), 171-219.

24. F. TRICERrI and L. VANHECKE, Homogeneous Structures on Riemannian Manifolds (London Mathematical Society Lecture Notes Series 83, Cambridge University Press, Cambridge, 1983).

25. H. WeYL, Classical groups, their invariants and representations (Princeton University Press, Princeton, 1946).

Dipartimento di Matematica

UNIVERSITÁ DI TORINO

via Carlo Alberto, 10

I-10123 TORINO

e-mail console@dm.unito.it / fino@dm.unito.it 